\title{
Parametric Extension of the Most Preferred OWA Operator and Its Application in Search Engine's Rank
}

\author{
Xiuzhi Sang and Xinwang Liu \\ School of Economics and Management, Southeast University, Nanjing, Jiangsu 210096, China \\ Correspondence should be addressed to Xiuzhi Sang; hellen1280@hotmail.com
}

Received 29 January 2013; Accepted 24 April 2013

Academic Editor: T. Warren Liao

Copyright ( 2013 X. Sang and X. Liu. This is an open access article distributed under the Creative Commons Attribution License, which permits unrestricted use, distribution, and reproduction in any medium, provided the original work is properly cited.

\begin{abstract}
Most preferred ordered weighted average (MP-OWA) operator is a new kind of neat (dynamic weight) OWA operator in the aggregation operator families. It considers the preferences of all alternatives across the criteria and provides unique aggregation characteristics in decision making. In this paper, we propose the parametric form of the MP-OWA operator to deal with the uncertainty preference information, which includes MP-OWA operator as its special case, and it also includes the most commonly used maximum, minimum, and average aggregation operators. A special form of parametric MP-OWA operator with power function is proposed. Some properties of the parametric MP-OWA operator are provided and the advantages of them in decision making problems are summarized. The new proposed parametric MP-OWA operator can grasp the subtle preference information of the decision maker according to the application context through multiple aggregation results. They are applied to rank search engines considering the relevance of the retrieved queries. An search engine ranking example illustrates the application of parametric MP-OWA operator in various decision situations.
\end{abstract}

\section{Introduction}

The ordered weighted averaging (OWA) operator, which was introduced by Yager [1], provides for aggregation lying between maximum and minimum operators and has received more and more attention since its appearance $[2,3]$. The OWA operator has been used in a wide range of applications, such as neural networks $[4,5]$, database systems $[6,7]$, fuzzy logic controllers $[8,9]$, decision making [10-12], expert systems [13-15], database query management and data mining $[16,17]$, and lossless image compression $[18,19]$.

Until now, according to the weight assignment methods, the existing OWA operators can be classified into two categories: one is the static OWA operators having weights depending on the serial position, and the other is dynamic or neat OWA operators having weights depending on the aggregated elements. The static OWA operators include the maximum entropy operator [20], minimum variance operator [21], the maximum Rényi entropy operator [22], least square deviation operator and chi-square operator [23], exponential OWA operator [24], linguistic ordered weighted averaging operator $[5,25]$, and intuitionistic fuzzy ordered weighted distance operator [26-28].
For neat OWA operator with dynamic weights, Yager $[29,30]$ proposed the families of neat OWA operator called basic defuzzification distribution (BADD) OWA operator and parameterized and-like and or-like OWA operators. Marimin et al. [31, 32] used neat OWA operator to aggregate the linguistic labels for expressing fuzzy preferences in group decision making problem. Peláez and Doña $[33,34]$ introduced majority additive OWA (MA-OWA) operator and quantified MA-OWA (QMA-OWA) operator to model the majority idea in group decision making problem. Liu and Lou [35] extended BADD OWA operator to additive neat OWA (ANOWA) operator for decision making problem. Wu et al. [36] introduced an argument-dependent approach based on maximizing the entropy.

Recently, Emrouznejad [3] proposed a new kind of neat OWA operator called most preferred OWA (MP-OWA) operator, which considers the preferences of alternatives across all the criteria. It has an interesting characteristic that the aggregation combines static OWA operator with dynamic OWA operator together. That is, because the weights correlate with internal aggregated elements in the way of neat OWA operator, and the aggregated elements must be ordered decreasingly when aggregating, which is the same as that 
of static-weight OWA operator, consequently, the MP-OWA operator not only has the advantage of neat OWA operator in which the weighting vector is relevant with the aggregated elements values rather than the positions, but also utilizes the most preferred information, which is connected with the maximum frequency of all scales for each criteria. Some extension researches about MP-OWA operator and the application can be found in the literature $[3,4,11,37,38]$.

In this paper, we propose parametric MP-OWA operator families, which combine the characteristics of MP-OWA operator with ordinary neat OWA operator together. We also propose the family of parametric MP-OWA operator with power function; it is quite useful as it includes the current MP-OWA operator as a special case and also includes multiple situations because of the aggregation results ranging between the minimum and the maximum. Meanwhile, some properties of the parametric MP-OWA operator and the MP-OWA operator family with power function are provided and analyzed, which can be used as the basis to apply our new parametric MP-OWA operator in practice. Moreover, we discuss the advantages of our new parametric MPOWA operator, which not only helps decision makers realize viewing the decision making problem completely through considering the preference relation and the parameter $(r)$, but also offers another kind of method for decision making problems based on preference information. We apply the proposed method to decision making problem concentrated on ranking search engines and get different rankings through changing the values of parameter $(r)$, which can help decision makers recognize the best search engines indirectly as well. It is necessary to stress that the proposed method can develop an amazingly wide range of decision making problems with preference relations such as information aggregation and group decision making.

This paper is organized as follows: Section 2 reviews some basic concepts of neat OWA operator and MP-OWA operator. Section 3 gives a general form of parametric MP-OWA operator and develops a particular member of MP-OWA operator with power function; some properties and advantages are also discussed. Section 4 gives an example of ranking search engines using the proposed approach. Section 5 summarizes the main results and draws conclusions.

\section{Preliminaries}

2.1. Neat OWA Operator. Yager [29] proposed neat OWA operator, which means the weighting vector not only depends on position indexes of the aggregated elements, but also the aggregated values.

Assume $x_{1}, x_{2}, \ldots, x_{n}$ is a collection of numbers; the aggregation of neat OWA operator is indicated as follows:

$$
F\left(x_{1}, x_{2}, \ldots, x_{n}\right)=\sum_{i=1}^{n} w_{i} y_{i},
$$

where $y_{i}$ is the $i$ th largest value of $x_{i}$ and $w_{i}$ is the weights to be a function of the ordered aggregated elements $y_{i}$, which is denoted as follows:

$$
w_{i}=f_{i}\left(y_{1}, y_{2}, \ldots, y_{n}\right) .
$$

The weights are required to satisfy two conditions:

(1) $w_{i} \in[0,1]$ for each $i$,

(2) $\sum_{i=1}^{n} w_{i}=1$.

In this case, (1) can be rewritten as follows:

$$
F\left(x_{1}, x_{2}, \ldots, x_{n}\right)=\sum_{i=1}^{n} f_{i}\left(y_{1}, y_{2}, \ldots, y_{n}\right) y_{i} .
$$

If $X=\left(x_{1}, x_{2}, \ldots, x_{n}\right)$ are inputs, $Y=\left(y_{1}, y_{2}, \ldots, y_{n}\right)$ are inputs ordered, and $Z=\left(z_{1}, z_{2}, \ldots, z_{n}\right)=\operatorname{Perm}\left(x_{1}, x_{2}, \ldots\right.$, $\left.x_{n}\right)$ is any permutation of the inputs, then the OWA operator is neat if

$$
F\left(x_{1}, x_{2}, \ldots, x_{n}\right)=\sum_{i=1}^{n} w_{i} y_{i}
$$

is the same for the assignment $Z=Y$.

Later, Yager and Filev [30] introduced the first family of neat OWA operator namely BADD OWA operator. The weighting vector is another collection of elements $v_{i}(i=$ $1,2, \ldots, n)$, such that

$$
v_{i}=\frac{x_{i}^{\alpha}}{\sum_{j=1}^{n} x_{j}^{\alpha}},
$$

where $\alpha \in[0,+\infty)$. It can be easily seen that BADD OWA operator has properties as follows:

(1) $v_{i} \in[0,1]$ for each $i$,

(2) $\sum_{i=1}^{n} v_{i}=1$.

From (5), the weighting vector of BADD OWA operator is expressed as follows:

$$
\begin{aligned}
W(\alpha) & =\left(w_{1}, w_{2}, \ldots, w_{n}\right)^{T} \\
& =\left(\frac{x_{1}^{\alpha}}{\sum_{j=1}^{n} x_{j}^{\alpha}}, \frac{x_{2}^{\alpha}}{\sum_{j=1}^{n} x_{j}^{\alpha}}, \ldots, \frac{x_{n}^{\alpha}}{\sum_{j=1}^{n} x_{j}^{\alpha}}\right)^{T} .
\end{aligned}
$$
follows:

Accordingly, the aggregation expression is denoted as

$$
F\left(x_{1}, x_{2}, \ldots, x_{n}\right)=\sum_{i=1}^{n} w_{i} x_{i}=\frac{\sum_{i=1}^{n} x_{i}^{\alpha+1}}{\sum_{j=1}^{n} x_{j}^{\alpha}} .
$$

Liu [39] proposed a generalization BADD OWA operator with weighted functional average, which is also called additive neat OWA (ANOWA) operator, where

$$
\begin{aligned}
W_{f}= & \left(w_{1}, w_{2}, \ldots, w_{n}\right)^{T} \\
= & \left(\frac{f\left(x_{1}\right)}{\sum_{j=1}^{n} f\left(x_{j}\right)}, \frac{f\left(x_{2}\right)}{\sum_{j=1}^{n} f\left(x_{j}\right)}, \ldots, \frac{f\left(x_{n}\right)}{\sum_{j=1}^{n} f\left(x_{j}\right)}\right)^{T}, \\
& F_{f}\left(x_{1}, x_{2}, \ldots, x_{n}\right)=\sum_{i=1}^{n} w_{i} x_{i}=\frac{\sum_{i=1}^{n} f\left(x_{i}\right) x_{i}}{\sum_{j=1}^{n} f\left(x_{j}\right)},
\end{aligned}
$$

where $f\left(x_{i}\right)$ can be any form of a continuous function. When $f\left(x_{i}\right)$ takes the form of power function, that is $f\left(x_{i}\right)=x_{i}^{\alpha}$, it turns into BADD OWA operator. 
2.2. The MP-OWA Operator. The MP-OWA operator, which was proposed by Emrouznejad [3], is based on the most popular criteria for all alternatives and considers the preferences of alternatives across all criteria. Suppose $Z=$ $\left\{Z_{1}, Z_{2}, \ldots, Z_{m} ; m \geqslant 2\right\}$ is a set of alternatives to be ranked, $C=\left\{C_{1}, C_{2}, \ldots, C_{n} ; n \geqslant 2\right\}$ is a group of criteria to rate the alternatives, $S=\left\{S_{1}, S_{2}, \ldots, S_{r}\right\}$ satisfying $S_{1}<S_{2}<\cdots<S_{r}$ is a given scale set, and $S_{j i} \in S$ is the scale value of alternative $A_{j}$ for criteria $C_{i}$. Then, the matrix of preference rating given to alternatives for each criteria is shown in Table 1.

Meanwhile, the frequency $N_{k i}(k \in[1, r], i \in[1, n])$ of scale $S_{k}$ given to criteria $C_{i}$ is summarized in Table 2 .

The frequency of the most popular scale for each criteria can be written as follows:

$$
\begin{aligned}
V= & \left(v_{1}, v_{2}, \ldots, v_{n}\right)^{T} \\
= & \left(\max \left\{N_{k 1}: \forall k\right\}, \max \left\{N_{k 2}: \forall k\right\}, \ldots,\right. \\
& \left.\quad \max \left\{N_{k n}: \forall k\right\}\right)^{T} .
\end{aligned}
$$

Accordingly, the weighting vector of MP-OWA operator can be expressed as follows:

$$
\begin{aligned}
W & =\left(w_{1}, w_{2}, \ldots, w_{n}\right)^{T} \\
& =\left(\frac{v_{1}}{\sum_{k=1}^{n} v_{k}}, \frac{v_{2}}{\sum_{k=1}^{n} v_{k}}, \ldots, \frac{v_{n}}{\sum_{k=1}^{n} v_{k}}\right)^{T} ;
\end{aligned}
$$

of course, $\sum_{i=1}^{n} w_{i}=1$.

The aggregation is expressed as follows:

$$
F_{W}\left(Z_{k}\right)=F_{W}\left(z_{k 1}, z_{k 2}, \ldots, z_{k n}\right)=\sum_{i=1}^{n} w_{i} y_{k i},
$$

where $y_{k i}$ is the $i$ th largest value of $z_{k i}$.

From (11), it is clear that the weight is independent of the ordering of set $V$; the more frequency of $S_{k}$ given to criteria $C_{j}$ is, the bigger the corresponding weight is. That is, the MPOWA operator overemphasizes the opinions of the majority and ignores those of the minority.

\section{Parametric MP-OWA Operator}

In this section, we firstly propose the general form of parametric MP-OWA operator, and some propositions are proposed. Then, we develop a particular family of parametric MP-OWA operator with power function, and some properties are also discussed.

3.1. The General Form of Parametric MP-OWA Operator. Similar to the extensions of OWA operator to the parametric form of BADD operator and ANOWA operator [30, 39], we will extend the MP-OWA operator to a parametric format, that can represent the preference information more flexibly, and MP operator becomes a special case of it.
TABLE 1: Matrix of preference rating of $n$ criteria with $m$ alternatives.

\begin{tabular}{ccccccc}
\hline & & \multicolumn{5}{c}{ Criteria } \\
& & $C_{1}$ & $\ldots$ & $C_{i}$ & $\ldots$ & $C_{n}$ \\
\hline \multirow{4}{*}{ Alternatives } & $Z_{1}$ & $S_{11}$ & $\ldots$ & $S_{1 i}$ & $\cdots$ & $S_{1 n}$ \\
& $\vdots$ & $\vdots$ & $\vdots$ & $\vdots$ & $\vdots$ & $\vdots$ \\
& $Z_{j}$ & $S_{j 1}$ & $\ldots$ & $S_{j i}$ & $\ldots$ & $S_{j n}$ \\
& $\vdots$ & $\vdots$ & $\vdots$ & $\vdots$ & $\vdots$ & $\vdots$ \\
& $Z_{m}$ & $S_{m 1}$ & $\ldots$ & $S_{m i}$ & $\cdots$ & $S_{m n}$ \\
\hline
\end{tabular}

TABLE 2: Matrix of frequency that scale gives to criteria.

\begin{tabular}{ccccccc}
\hline & & \multicolumn{5}{c}{ Criteria } \\
& $C_{1}$ & $\ldots$ & $C_{i}$ & $\ldots$ & $C_{n}$ \\
\hline \multirow{4}{*}{ Scales } & $S_{1}$ & $N_{11}$ & $\ldots$ & $N_{1 i}$ & $\ldots$ & $N_{1 n}$ \\
& $\vdots$ & $\vdots$ & $\vdots$ & $\vdots$ & $\vdots$ & $\vdots$ \\
& $S_{k}$ & $N_{k 1}$ & $\ldots$ & $N_{k i}$ & $\ldots$ & $N_{k n}$ \\
& $\vdots$ & $\vdots$ & $\vdots$ & $\vdots$ & $\vdots$ & $\vdots$ \\
& $S_{r}$ & $N_{r 1}$ & $\ldots$ & $N_{r i}$ & $\ldots$ & $N_{r n}$ \\
\hline
\end{tabular}

Definition 1. For aggregated matrix $Z=\left(Z_{1}, Z_{2}, \ldots, Z_{m}\right)$, $Z_{j}=\left(z_{j 1}, z_{j 2}, \ldots, z_{j n}\right)(j \in[1, m]) . v_{i}=\max \left\{N_{k i}, k \in\right.$ $[1, r]\}(i \in[1, n])$, and $f\left(v_{i}\right) \geqslant 0$, where $N_{k i}$ is the frequency of each scale for criteria. The vector of the maximum frequency function can be written as follows:

$$
V_{f}=\left(f\left(v_{1}\right), f\left(v_{2}\right), \ldots, f\left(v_{n}\right)\right)^{T} .
$$

The weighting vector is defined as follows:

$$
\begin{aligned}
W_{f} & =\left(w_{1}, w_{2}, \ldots, w_{n}\right)^{T} \\
& =\left(\frac{f\left(v_{1}\right)}{\sum_{j=1}^{n} f\left(v_{j}\right)}, \frac{f\left(v_{2}\right)}{\sum_{j=1}^{n} f\left(v_{j}\right)}, \ldots, \frac{f\left(v_{n}\right)}{\sum_{j=1}^{n} f\left(v_{j}\right)}\right)^{T} .
\end{aligned}
$$

Here, $f\left(v_{i}\right)$ can be substituted for many specific functions.

It is obvious that $w_{i}$ satisfies the normalization properties of $w_{i} \geqslant 0$ and $\sum_{i=1}^{n} w_{i}=1$.

The parametric MP-OWA operator aggregation is

$$
F_{f}(Z)=\sum_{j=1}^{m} \sum_{i=1}^{n} w_{i} y_{j i}=\sum_{j=1}^{m} \sum_{i=1}^{n} \frac{f\left(v_{i}\right)}{\sum_{j=1}^{n} f\left(v_{j}\right)} y_{j i},
$$

where $y_{j i}$ is the $i$ th largest value of $z_{j i}$.

In (14), if $f\left(v_{i}\right)=v_{i}(i \in[1, n]),(15)$ is the same as (12); that is, MP-OWA operator becomes a special case of the parametric MP-OWA operator.

Next, we will give some properties of our new proposed parametric MP-OWA operator.

Definition 2. Assume $F_{f}$ is a parametric MP-OWA operator with a weighting vector $W_{f}$; the degree of orness $\left(W_{f}\right)$ is defined as follows:

$$
\operatorname{orness}(W)=\sum_{i=1}^{n} \frac{n-i}{n-1} \frac{f\left(v_{i}\right)}{\sum_{j=1}^{n} f\left(v_{j}\right)} .
$$


Next, some propositions of the parametric MP-OWA operator are described as:

Proposition 3. Assume $F_{f}$ is the aggregation result with parametric MP-OWA operator and $f\left(v_{i}\right)$ is the ith value of the set $V$.

(1) Boundary. If $X=\left(x_{1}, x_{2}, \ldots, x_{n}\right)$ is the aggregated elements, then

$\min _{1 \leqslant i \leqslant n}\left\{f\left(x_{i}\right)\right\} \leqslant F_{f}\left(x_{1}, x_{2}, \ldots, x_{n}\right) \leqslant \max _{1 \leqslant i \leqslant n}\left\{f\left(x_{i}\right)\right\}$.

(2) Commutativity. If $x_{i}$ and $x_{i}^{(k)}$ are the ith largest values of the aggregated sets $X$ and $X^{K}$, respectively, then

$F_{f}\left(x_{1}, x_{2}, \ldots, x_{n}\right)=F_{f}\left(x_{1}^{(k)}, x_{2}^{(k)}, \ldots, x_{n}^{(k)}\right)$,

where $\left(x_{1}^{(k)}, x_{2}^{(k)}, \ldots, x_{n}^{(k)}\right)$ is any permutation of the arguments $\left(x_{1}, x_{2}, \ldots, x_{n}\right)$.

(3) Monotonicity. If $x_{i}$ and $y_{i}$ are the ith largest values of the aggregated sets $X$ and $Y$, respectively, and $y_{i} \leqslant x_{i}$, then

$$
F\left(y_{1}, y_{2}, \ldots, y_{n}\right) \leqslant F\left(x_{1}, x_{2}, \ldots, x_{n}\right),
$$

where the vector $V$ is the same as both aggregated vectors.

(4) Idempotency. If $X=\left(x_{1}, x_{2}, \ldots, x_{n}\right)$ is the aggregated elements, and $x_{i}=x(i=1,2, \ldots, n)$, then

$$
F\left(x_{1}, x_{2}, \ldots, x_{n}\right)=x \text {. }
$$

Apparently, if we set $f\left(v_{i}\right)=v_{i}(i \in[1, n])$, parametric MP-OWA operator turns into MP-OWA operator, and the conclusions of Proposition 3 are also correct.

Proposition 4. Assume $F$ is the MP-OWA operator aggregation result and $v_{i}$ is the ith value of set $V$.

(1) Boundary. If $X=\left(x_{1}, x_{2}, \ldots, x_{n}\right)$ is the aggregated elements, then

$$
\min _{1 \leqslant i \leqslant n}\left\{x_{i}\right\} \leqslant F\left(x_{1}, x_{2}, \ldots, x_{n}\right) \leqslant \max _{1 \leqslant i \leqslant n}\left\{x_{i}\right\} .
$$

(2) Commutativity. If $x_{i}$ and $x_{i}^{(k)}$ are the ith largest values of the aggregated sets $X$ and $X^{K}$, respectively, then

$$
F\left(x_{1}, x_{2}, \ldots, x_{n}\right)=F\left(x_{1}^{(k)}, x_{2}^{(k)}, \ldots, x_{n}^{(k)}\right),
$$

where $\left(x_{1}^{(k)}, x_{2}^{(k)}, \ldots, x_{n}^{(k)}\right)$ is any permutation of the arguments $\left(x_{1}, x_{2}, \ldots, x_{n}\right)$.

(3) Monotonicity. If $x_{i}$ and $y_{i}$ are the ith largest values of the aggregated sets $X$ and $Y$, respectively, and $y_{i} \leqslant x_{i}$, for each $i(i=1,2, \ldots, n)$, then

$$
F\left(y_{1}, y_{2}, \ldots, y_{n}\right) \leqslant F\left(x_{1}, x_{2}, \ldots, x_{n}\right),
$$

where $V$ is the same vector as both aggregated values of $y_{i}$ and $x_{i}$.

(4) Idempotency. If $X=\left(x_{1}, x_{2}, \ldots, x_{n}\right)$ is the aggregated elements, and $x_{i}=x(i=1,2, \ldots, n)$, then

$$
F\left(x_{1}, x_{2}, \ldots, x_{n}\right)=x \text {. }
$$

3.2. Parametric MP-OWA Operator with Power Function. Similar to ANOWA operator (8), which takes the form of power function and becomes BADD OWA operator, we study the family of parametric MP-OWA operator with power function, and the function $f\left(v_{i}\right)$ is given in the following form as:

$$
f\left(v_{i}\right)=v_{i}^{r},
$$

where $r$ is a real number.

From (14), the weighting vector of parametric MP-OWA operator can be rewritten as follows:

$$
\begin{aligned}
W_{f} & =\left(\frac{f\left(v_{1}\right)}{\sum_{j=1}^{n} f\left(v_{j}\right)}, \frac{f\left(v_{2}\right)}{\sum_{j=1}^{n} f\left(v_{j}\right)}, \ldots, \frac{f\left(v_{n}\right)}{\sum_{j=1}^{n} f\left(v_{j}\right)}\right)^{T} \\
& =\left(\frac{v_{1}^{r}}{\sum_{j=1}^{n} v_{j}^{r}}, \frac{v_{2}^{r}}{\sum_{j=1}^{n} v_{j}^{r}}, \ldots, \frac{v_{n}^{r}}{\sum_{j=1}^{n} v_{j}^{r}}\right)^{T} .
\end{aligned}
$$

Accordingly, from (15), the aggregation can be expressed as follows:

$$
F_{f}\left(x_{1}, x_{2}, \ldots, x_{n}\right)=\sum_{i=1}^{n} w_{i} y_{i}=\sum_{i=1}^{n} \frac{v_{i}^{r}}{\sum_{j=1}^{n} v_{j}^{r}} y_{i},
$$

where $y_{i}$ is the $i$ th largest value of $x_{i}$.

Regarding (16), the orness equation can also be described as follows:

$$
\operatorname{orness}\left(W_{f}\right)=\sum_{i=1}^{n} \frac{n-i}{n-1} w_{i}=\sum_{i=1}^{n} \frac{n-i}{n-1} \frac{v_{i}^{r}}{\sum_{j=1}^{n} v_{j}^{r}},
$$

when $r=1$ in (25), parametric MP-OWA operator becomes ordinary MP-OWA operator.

Remark 5. Generally speaking, in the parametric MP-OWA operator (15), $f\left(v_{i}\right)$ can take any function forms, such as power function, exponential function, or other function forms. Here, we only take the form of power function. The reasons for this decision are as follows: (1) Power function for parametric MP-OWA operator with $f\left(v_{i}\right)=v_{i}^{\alpha}$ can deduce the ordinary MP-OWA operator very naturally with $\alpha=1$. But parametric MP-OWA operator with other forms cannot do it. (2) The parameter in power function and other functions does not have any common, which makes parametric MP-OWA operator different from both in expressions and final aggregation results, so that, they do not need to be put together and compared with each other. (3) Because we have extended the MP-OWA operator to the parametric format, we can compare the results on various parameter values. But the comparisons of both different function formats and different parameter values of each format will be complicated; neither much facts, nor much help to problem understanding can be observed.

From (13), the maximum frequency vector $V_{f}$ with power function can also be denoted as follows:

$$
\begin{aligned}
V_{f} & =\left(f\left(v_{1}\right), f\left(v_{2}\right), \ldots, f\left(v_{n}\right)\right)^{T} \\
& =\left(v_{1}^{r}, v_{2}^{r}, \ldots, v_{n}^{r}\right)^{T} .
\end{aligned}
$$


For parametric MP-OWA operator, $f\left(v_{i}\right)=v_{i}^{r}$ is a monotonic function with argument $v_{i}$. If parameter $r>0, f\left(v_{i}\right)$ increases with $v_{i}$. With the increasing of $v_{i}$, larger (smaller) aggregated elements will be given more (less) emphasis. If $r<0, f\left(v_{i}\right)$ decreases with $v_{i}$. With the increasing of $v_{i}$, larger (smaller) aggregated elements will be given less (more) emphasis.

Therefore, if the decision maker wants to put more emphasis on large aggregated elements and less emphasis on small aggregated elements, he or she can choose $r>0$; if he or she wants to put more emphasis on small aggregated elements and less emphasis on large elements, $r<0$ can be selected.

Some properties of parametric MP-OWA operator with power function $f\left(v_{i}\right)=v_{i}^{r}$ are discussed in the following.

Theorem 6. Assume $F_{f}$ is the parametric MP-OWA operator, $f\left(v_{i}\right)=v_{i}^{r}$ is the ith value of set $V$ and $x_{i}$ is the ith value of set $X$.

(1) For $r \rightarrow-\infty$, the orness is $(n-k) /(n-1)$, and $F_{f}(X)=((n-k) /(n-1)) x_{k}$, where $k$ is the index of the $\min _{1 \leqslant i \leqslant n}\left\{v_{i}\right\}$.

(2) For $r=0$, the orness is $1 / 2$, and $F_{f}(X)=\operatorname{avg}\left\{x_{i}\right\}$.

(3) For $r \rightarrow+\infty$, the orness is $(n-l) /(n-1)$, and $F_{f}(X)=((n-l) /(n-1)) x_{l}$, where $l$ is the index of the $\max _{1 \leqslant i \leqslant n}\left\{v_{i}\right\}$.

Proof. See Appendix A.

Remark 7. By using different values of parameter $r$ for parametric MP-OWA operator, people can get different weighting vectors for decision making. For example, if the decision makers have no subjective preference for aggregated elements, they can select $r=0$ or MP-OWA operator. If they want to underweight large aggregated elements and overweight small aggregated elements, parameter $r<0$ is the right choice; when the parameter $r$ decreases to a certain negative number, the weights according to large aggregated elements reach zero; that is, the decision makers would neglect the influence of large aggregated elements and stress the small elements to the ultimate aggregation results. On the contrary, they can choose $r>0$.

Theorem 8. Assume $F_{f}$ is the parametric MP-OWA operator, $f\left(v_{i}\right)$ is the ith values of the set $V$, and $f\left(v_{i}\right)=v_{i}^{r}(i \in[1, n])$. If $r_{1}>r_{2}$, then orness $\left(W_{r_{1}}\right)>\operatorname{orness}\left(W_{r_{2}}\right)$.

Proof. See Appendix B.

3.3. Advantages of the Parametric MP-OWA Operator in Decision Making. Compared with the MP-OWA operator, the advantages of the parametric MP-OWA operator are summarized as follows:

(1) It extends the MP-OWA operator to a parametric form, which brings about more flexibility in practice. The parametric MP-OWA operator can generate multiple weighting vectors through changing the values of the parameter $r$; people may select appropriate weighting vector to reflect their preferences, which provide more flexibility for decision making. However, the MP-OWA operator obtains merely one weighting vector, which does not reflect any attitude of the decision makers to the aggregated elements, and people could not change the ultimate aggregation result any more.

(2) It provides a power function as a specific form to compute the weighting vector. Decision makers can choose different values of parameter $r$ according to their interest and actual application context.

(3) It offers another kind of method for problems concentrated on ranking search engines. Parametric MPOWA operator is based on the use of multiple decision making process, where a group of queries retrieved from selected search engines are used to look for an optimal ranking of the search engines. It can also identify which are the best search engines at the same time.

(4) It is necessary to stress that the proposed method can develop an amazingly wide range of decision making problems with preference relations, such as information aggregation and group decision making.

\section{The Application of Parametric MP-OWA Operator in Ranking Internet Search Engine Results}

4.1. Background. Emrouznejad [3] used OWA operator to measure the performance of search engines by factors such as average click rate, total relevancy of the returned results, and the variance of the clicked results. In their study, a number of students were asked to execute the sample search queries. They classified the results into three categories: relevant, undecided, and irrelevant documents, whose values are 2, 1 , and 0 , respectively. Each participant was asked to evaluate the result items and the results are shown denoted as matrix $Z$ in Table 3.

The frequencies of all scales for each query are shown in Table 4 .

4.2. Computing Process. To further understand what the influence of parametric MP-OWA operator on the results of decision making will be, the weighting vectors, aggregation results, and ranking lists are computed and compared with the MP-OWA operator.

From (10), it is obvious that the maximum frequency of each query in Table 4 is

$$
V=(9,7,5,8,6,5,4,7,6,4,6,6)^{T} .
$$

Next, we will use $r=-4,-3,-2,-1,0,1,2,3,4$ of power function for parametric MP-OWA operator to rank the search engines, and the ranks are compared with those of MP-OWA operator. Take $r=2$, for example; the computing process is as follows.

From (25), we get

$$
f\left(v_{i}\right)=v_{i}^{2}, \quad i \in[1,12],
$$


TABLE 3: Matrix of judgment for sample queries.

\begin{tabular}{|c|c|c|c|c|c|c|c|c|c|c|c|c|}
\hline Queries/search engines & 1 & 2 & 3 & 4 & 5 & 6 & 7 & 8 & 9 & 10 & 11 & 12 \\
\hline LookSmart & 2 & 1 & 0 & 2 & 0 & 2 & 0 & 2 & 0 & 0 & 2 & 1 \\
\hline Lycos & 2 & 1 & 0 & 2 & 1 & 1 & 2 & 2 & 0 & 1 & 1 & 2 \\
\hline Altavista & 2 & 2 & 1 & 2 & 1 & 0 & 2 & 1 & 2 & 2 & 1 & 0 \\
\hline Msn & 2 & 1 & 2 & 0 & 0 & 2 & 1 & 2 & 2 & 1 & 1 & 2 \\
\hline Yahoo & 1 & 2 & 2 & 2 & 1 & 1 & 0 & 0 & 2 & 2 & 1 & 1 \\
\hline Teoma & 2 & 2 & 0 & 1 & 1 & 2 & 0 & 2 & 2 & 2 & 1 & 0 \\
\hline WiseNut & 2 & 1 & 2 & 2 & 1 & 0 & 1 & 2 & 2 & 0 & 0 & 0 \\
\hline MetaCrawler & 1 & 2 & 0 & 2 & 2 & 2 & 0 & 2 & 0 & 1 & 2 & 2 \\
\hline ProFusion & 2 & 2 & 2 & 0 & 1 & 1 & 2 & 2 & 2 & 0 & 1 & 2 \\
\hline WebFusion-Max & 2 & 2 & 1 & 2 & 0 & 2 & 2 & 1 & 1 & 1 & 2 & 2 \\
\hline WebFusion-OWA & 2 & 2 & 2 & 2 & 2 & 1 & 1 & 1 & 1 & 2 & 2 & 2 \\
\hline
\end{tabular}

TABLE 4: The frequencies of all scales for each query.

\begin{tabular}{lllllllllllll}
\hline Queries/scales & 1 & 2 & 3 & 4 & 5 & 6 & 7 & 8 & 9 & 10 & 11 \\
\hline 0 & 0 & 0 & 4 & 2 & 3 & 2 & 4 & 1 & 3 & 3 & 1 & 3 \\
1 & 2 & 4 & 2 & 1 & 6 & 4 & 3 & 3 & 2 & 4 & 4 & 2 \\
2 & 9 & 7 & 5 & 8 & 2 & 5 & 4 & 7 & 6 & 4 & 6 & 6 \\
\hline
\end{tabular}

From (29), the maximum frequency vector with power function is

$$
\begin{aligned}
V_{f(r=2)} & =\left(v_{1}^{2}, v_{2}^{2}, \ldots, v_{12}^{2}\right)^{T} \\
& =(81,49,25,64,36,25,16,49,36,16,36,36)^{T} .
\end{aligned}
$$

Take the result $V_{f(r=2)}$ into (26); obtain the corresponding weighting vector $W_{f}$ as follows:

$$
\begin{aligned}
W_{f(r=2)}= & \left(\frac{f\left(v_{1}\right)}{\sum_{j=1}^{12} f\left(v_{j}\right)}, \frac{f\left(v_{2}\right)}{\sum_{j=1}^{12} f\left(v_{j}\right)}, \ldots, \frac{f\left(v_{12}\right)}{\sum_{j=1}^{12} f\left(v_{j}\right)}\right)^{T} \\
= & (0.17,0.10,0.05,0.14,0.08,0.05,0.03,0.10 \\
& 0.08,0.03,0.08,0.08)^{T} .
\end{aligned}
$$

Take the result $W_{f(r=2)}$ into (33) and the matrix $Z$ of Table 3 into (27); the aggregation result is

$$
\begin{aligned}
F_{f(r=2)}= & Z W_{f} \\
= & (1.17,1.39,1.44,1.44,1.39,1.41, \\
& 1.28,1.44,1.48,1.55,1.74)^{T} .
\end{aligned}
$$

It is noticed that matrix $Z$ of Table 3 must be ordered decreasingly in each row before information aggregation.

With the same method, we get other aggregation results with parameter $r=-4,-3,-2,-1,0,1,3,4$, which are displayed in Table 5, the last column of which is calculated with the MP-OWA operator by Emrouznejad [3].
Correspondingly, the aggregation results of parametric MP-OWA operator with parameter $r=-4,-3,-2,-1,0,1$, 2,3, 4 and MP-OWA operator are listed in Table 6. And the ranks given to each search engine using parametric MP-OWA operator with power function and MP-OWA operator are shown in Table 7.

\subsection{Comparisons and Some Discussions}

(1) From Table 5, it is seen that if $r>0$, the larger (smaller) of the values $r$ are, the larger (smaller) of the values $f\left(v_{i}\right)$ are, and the weights of search engines become larger (smaller) correspondingly. That is, more (less) emphasis would be put on larger (smaller) aggregated elements. For example, no matter $r=$ $4,3,2$, or $1, v_{1}=9$ has the largest weights, whereas $v_{4}=v_{7}=4$ has the smallest weights.

(2) When $r=4, w_{10}=0.01$; that is, there is almost no emphasis put on the smallest aggregated element. As the monotonicity of function $f\left(v_{i}\right)$ with $v_{i}$, if $r$ continues to increase, there will appear more zero weights, and the aggregation results may lose more information.

(3) If $r \leqslant 0$, the larger (smaller) of the values $v_{i}$ are, the smaller (larger) of the values $f\left(v_{i}\right)$ are, and the weights of search engines become smaller (larger) correspondingly. That is, more (less) emphasis would be put on smaller (bigger) aggregated elements. For example, no matter what $r=0,-1,-2,-3$, or -4 , $v_{1}=9$ has the smallest weights, whereas $v_{4}=v_{7}=4$ has the largest weights.

(4) When $r=-1, w_{1}=0$; that is, there is no emphasis put on the largest aggregated element. As the monotonicity of function $f\left(v_{i}\right)$ with $v_{i}$, if $r$ continues to 
TABLE 5: Weights given to search engines with different values of parameter $r$.

\begin{tabular}{|c|c|c|c|c|c|c|c|c|c|c|c|}
\hline \multirow{2}{*}{ Methods/weights } & \multicolumn{10}{|c|}{ Parametric Mp-OWA operator parameter $r$} & \multirow{2}{*}{ MP-OWA operator } \\
\hline & $V_{0}$ & -4 & -3 & -2 & -1 & 0 & 1 & 2 & 3 & 4 & \\
\hline$w_{1}$ & 9 & 0.01 & 0.02 & 0.03 & 0.05 & 0.08 & 0.12 & 0.17 & 0.23 & 0.29 & 0.12 \\
\hline$w_{2}$ & 7 & 0.03 & 0.04 & 0.05 & 0.07 & 0.08 & 0.10 & 0.10 & 0.11 & 0.11 & 0.10 \\
\hline$w_{3}$ & 5 & 0.10 & 0.11 & 0.10 & 0.10 & 0.08 & 0.07 & 0.05 & 0.04 & 0.03 & 0.07 \\
\hline$w_{4}$ & 8 & 0.02 & 0.03 & 0.04 & 0.06 & 0.08 & 0.11 & 0.14 & 0.16 & 0.18 & 0.11 \\
\hline$w_{5}$ & 6 & 0.05 & 0.06 & 0.07 & 0.08 & 0.08 & 0.08 & 0.08 & 0.07 & 0.06 & 0.08 \\
\hline$w_{6}$ & 5 & 0.10 & 0.11 & 0.10 & 0.10 & 0.08 & 0.07 & 0.05 & 0.04 & 0.03 & 0.07 \\
\hline$w_{7}$ & 4 & 0.25 & 0.21 & 0.16 & 0.12 & 0.08 & 0.05 & 0.03 & 0.02 & 0.01 & 0.05 \\
\hline$w_{8}$ & 7 & 0.03 & 0.04 & 0.05 & 0.07 & 0.08 & 0.10 & 0.10 & 0.11 & 0.11 & 0.10 \\
\hline$w_{9}$ & 6 & 0.05 & 0.06 & 0.07 & 0.08 & 0.08 & 0.08 & 0.08 & 0.07 & 0.06 & 0.08 \\
\hline$w_{10}$ & 4 & 0.25 & 0.21 & 0.16 & 0.12 & 0.08 & 0.05 & 0.03 & 0.02 & 0.01 & 0.05 \\
\hline$w_{11}$ & 6 & 0.05 & 0.06 & 0.07 & 0.08 & 0.08 & 0.08 & 0.08 & 0.07 & 0.06 & 0.08 \\
\hline$w_{12}$ & 6 & 0.05 & 0.06 & 0.07 & 0.08 & 0.08 & 0.08 & 0.08 & 0.07 & 0.06 & $\mathbf{0 . 0 8}$ \\
\hline
\end{tabular}

TABLE 6: Aggregation results given to search engines with different values of parameter $r$.

\begin{tabular}{|c|c|c|c|c|c|c|c|c|c|c|}
\hline \multirow{2}{*}{ Methods/search engines } & \multicolumn{9}{|c|}{ Parametric Mp-OWA operator with parameter $r$} & \multirow{2}{*}{ MP-OWA operator } \\
\hline & -4 & -3 & -2 & -1 & 0 & 1 & 2 & 3 & 4 & \\
\hline LookSmart & 0.77 & 0.82 & 0.87 & 0.93 & 1 & 1.08 & 1.17 & 1.27 & 1.38 & 1.08 \\
\hline Lycos & 1.1 & 1.13 & 1.16 & 1.2 & 1.25 & 1.32 & 1.39 & 1.47 & 1.55 & 1.32 \\
\hline Altavista & 1.21 & 1.24 & 1.26 & 1.29 & 1.33 & 1.38 & 1.44 & 1.51 & 1.58 & 1.38 \\
\hline MSN & 1.21 & 1.24 & 1.26 & 1.29 & 1.33 & 1.38 & 1.44 & 1.51 & 1.58 & 1.38 \\
\hline Yahoo & 1.1 & 1.13 & 1.16 & 1.2 & 1.25 & 1.32 & 1.39 & 1.47 & 1.55 & 1.32 \\
\hline Teoma & 0.95 & 1.03 & 1.1 & 1.17 & 1.25 & 1.33 & 1.41 & 1.49 & 1.57 & 1.33 \\
\hline WiseNut & 0.80 & 0.86 & 0.92 & 1 & 1.08 & 1.18 & 1.28 & 1.38 & 1.48 & 1.18 \\
\hline MetaCrawler & 1.21 & 1.24 & 1.26 & 1.29 & 1.33 & 1.38 & 1.44 & 1.51 & 1.58 & 1.38 \\
\hline ProFusion & 1.46 & 1.45 & 1.42 & 1.41 & 1.42 & 1.44 & 1.48 & 1.53 & 1.59 & 1.44 \\
\hline WebFusion-Max & 1.51 & 1.51 & 1.5 & 1.49 & 1.5 & 1.52 & 1.55 & 1.6 & 1.65 & 1.52 \\
\hline WebFusion-OWA & 1.59 & 1.61 & 1.62 & 1.64 & 1.67 & 1.70 & 1.74 & 1.78 & 1.82 & 1.70 \\
\hline
\end{tabular}

decrease, there will appear more zero weights, and the aggregation would lose more information.

(5) When $r=1$, the weights and the aggregation results are the same as those of MP-OWA operator, which are labeled in bold in Tables 5 and 6 .

It is shown that MP-OWA operator is a special case of parametric MP-OWA operator with function function on condition of $r=1$.

(6) Here, we only list a few values of parametric MP-OWA operator with power function, but we have included all the ranking with this method.

Because when $r>0$, although the weight and the aggregation results of each search engines both change steadily, the rank remains the same; when $r \leqslant$ 0 , the rank shows the similar regularity as well. In other words, with different values of parameter $r$, we get two kinds of aggregation results; the conditions are $r>0$ and $r \leqslant 0$.

(7) It can also be seen that the ranks of most search engines on each method keep the same, especially the WebFusion-OWA, WebFusion-Max, ProFusion, and LookSmart. It is noticeably that no matter what methods we use, search engines WebFusion-OWA, WebFusion-Max, ProFusion, and LookSmart remains in the first, second, and third place, respectively. From the result, we also deduce the best search engines indirectly.

\section{Conclusions}

We have presented a new kind of neat OWA operator based on MP-OWA operator in aggregation families when considering the decision maker's preference for all alternatives across the criteria. It is very useful for decision makers, since it not only considers the preference of alternatives across all the criteria, but also provides multiple aggregation results according to their preferences and application context to choose. We have discussed several properties and have studied particular cases such as minimum, average, and maximum aggregation results.

We have analyzed the applicability of parametric MPOWA operator that gets more comprehensive results than MP-OWA operator. We have concentrated on an application in ranking search engines based on multiple decision making 
TABLE 7: Comparison of ranks given to search engines with different values of parameter $r$.

\begin{tabular}{|c|c|c|c|c|c|c|c|c|c|c|}
\hline \multirow{2}{*}{ Methods/search engines } & \multicolumn{9}{|c|}{ Parametric Mp-OWA operator with parameter $r$} & \multirow{2}{*}{ MP-OWA operator } \\
\hline & -4 & -3 & -2 & -1 & 0 & 1 & 2 & 3 & 4 & \\
\hline WebFusion-OWA & 1 & 1 & 1 & 1 & 1 & 1 & 1 & 1 & 1 & 1 \\
\hline WebFusion-Max & 2 & 2 & 2 & 2 & 2 & 2 & 2 & 2 & 2 & 2 \\
\hline ProFusion & 3 & 3 & 3 & 3 & 3 & 3 & 3 & 3 & 3 & 3 \\
\hline Altavista & 4 & 4 & 4 & 4 & 4 & 4 & 4 & 4 & 4 & 4 \\
\hline Msn & 5 & 5 & 5 & 5 & 5 & 5 & 5 & 5 & 5 & 5 \\
\hline MetaCrawler & 6 & 6 & 6 & 6 & 6 & 6 & 6 & 6 & 6 & 6 \\
\hline Teoma & 9 & 9 & 9 & 9 & 9 & 7 & 7 & 7 & 7 & 7 \\
\hline Lycos & 7 & 7 & 7 & 7 & 7 & 8 & 8 & 8 & 8 & 8 \\
\hline Yahoo & 8 & 8 & 8 & 8 & 8 & 9 & 9 & 9 & 9 & 9 \\
\hline WiseNut & 10 & 10 & 10 & 10 & 10 & 10 & 10 & 10 & 10 & 10 \\
\hline LookSmart & 11 & 11 & 11 & 11 & 11 & 11 & 11 & 11 & 11 & 11 \\
\hline
\end{tabular}

process, where a group of queries are used to look for an optimal search engines list. And the decision makers can realize viewing the decision making problem completely through considering the preference relation and the corresponding parameter $r$. It is observed that no matter what values of the parameter $r$ are, the ranking of some search engines keeps the same. It also implies which the best search engines are. Finally, it should be noted that the proposed method can also be applied to a wide range of decision making problems with preference relations, such as information aggregation and group decision making.

In our future research, we expect to further propose parametric MP-OWA operator through employing other type of preference information such as linguistic variables and type-2 fuzzy set. We will develop different type of applications not only in decision theory but also in other fields such as engineering and economics.

\section{Appendices}

\section{A. Proof of Theorem 6}

Proof. (1) For $r \rightarrow-\infty$, we get

$$
\lim _{r \rightarrow-\infty} \frac{v_{i}^{r}}{v_{j}^{r}}=\lim _{r \rightarrow-\infty}\left(\frac{v_{i}}{v_{j}}\right)^{r}= \begin{cases}0, & \text { if } v_{i}>v_{j}, \\ 1, & \text { if } v_{i}=v_{j}, \\ +\infty, & \text { if } v_{i}<v_{j} .\end{cases}
$$

From (A.1), it is also right that

$$
\lim _{r \rightarrow-\infty}\left(\frac{v_{i}}{v_{j}}\right)^{-r}= \begin{cases}+\infty, & \text { if } v_{i}>v_{j}, \\ 1, & \text { if } v_{i}=v_{j}, \\ 0, & \text { if } v_{i}<v_{j} .\end{cases}
$$

Accordingly, from (A.2), when $n$ is a large integer, it is obvious that

$$
\lim _{r \rightarrow-\infty} \frac{v_{i}^{r}}{\sum_{j=1}^{n} v_{j}^{r}}=\lim _{r \rightarrow-\infty} \frac{1}{\sum_{j=1}^{n}\left(v_{i} / v_{j}\right)^{-r}}= \begin{cases}0, & \text { if } v_{i}>v_{j}, \\ 0, & \text { if } v_{i}=v_{j}, \\ 1, & \text { if } v_{i}<v_{j} .\end{cases}
$$

When $r \rightarrow-\infty$, combining (A.3) with (28), we obtain

$$
\begin{aligned}
\operatorname{orness}(W) & =\lim _{r \rightarrow-\infty} \sum_{i=1}^{n} \frac{n-i}{n-1} \frac{v_{i}^{r}}{\sum_{j=1}^{n} v_{j}^{r}} \\
& =\lim _{r \rightarrow-\infty} \sum_{i=1}^{n} \frac{n-i}{n-1} \frac{1}{\sum_{j=1}^{n}\left(v_{i} / v_{j}\right)^{-r}} \\
& =\frac{n-j}{n-1},
\end{aligned}
$$

where $j$ is the index of minimum $v_{i}$.

Accordingly, combine (A.3) with (27), and the aggregation result is

$$
F_{f}\left(x_{1}, x_{2}, \ldots, x_{n}\right)=\min \left\{x_{i}\right\}=x_{k},
$$

where $k$ is the index of minimum $x_{i}$.

(2) For $r=0$, from (28), we get

$$
\begin{aligned}
\operatorname{orness}(W) & =\sum_{i=1}^{n} \frac{n-i}{n-1} \frac{1}{\sum_{j=1}^{n}\left(v_{i} / v_{j}\right)^{-r}} \\
& =\sum_{i=1}^{n} \frac{n-i}{n-1} \frac{1}{n} \\
& =\frac{1}{2},
\end{aligned}
$$

such that from (27), the aggregation result is

$$
F_{f}\left(x_{1}, x_{2}, \ldots, x_{n}\right)=\sum_{i=1}^{n} \frac{1}{n} x_{i}=\operatorname{avg}\left\{x_{i}\right\} .
$$

(3) For $r \rightarrow+\infty$, we obtain

$$
\lim _{r \rightarrow+\infty} \frac{v_{i}^{r}}{v_{j}^{r}}=\lim _{r \rightarrow+\infty} \frac{1}{\left(v_{i} / v_{j}\right)^{r}}= \begin{cases}+\infty, & \text { if } v_{i}>v_{j}, \\ 1, & \text { if } v_{i}=v_{j}, \\ 0, & \text { if } v_{i}<v_{j} .\end{cases}
$$


From (A.8), it is also right that

$$
\lim _{r \rightarrow+\infty}\left(\frac{v_{i}}{v_{j}}\right)^{-r}= \begin{cases}0, & \text { if } v_{i}>v_{j} \\ 1, & \text { if } v_{i}=v_{j} \\ +\infty, & \text { if } v_{i}<v_{j}\end{cases}
$$

From the conclusion of (A.9), when $n$ is a large integer, it is obvious that

$$
\lim _{r \rightarrow+\infty} \frac{v_{i}^{r}}{\sum_{j=1}^{n} v_{j}^{r}}=\lim _{r \rightarrow+\infty} \frac{1}{\sum_{j=1}^{n}\left(v_{i} / v_{j}\right)^{-r}}= \begin{cases}1, & \text { if } v_{i}>v_{j}, \\ 0, & \text { if } v_{i}=v_{j}, \\ 0, & \text { if } v_{i}<v_{j} .\end{cases}
$$

Combining (28) with (A.10), the orness level is

$$
\begin{aligned}
\operatorname{orness}(W) & =\lim _{r \rightarrow+\infty} \sum_{i=1}^{n} \frac{n-i}{n-1} \frac{v_{i}^{r}}{\sum_{j=1}^{n} v_{j}^{r}}, \\
& =\lim _{r \rightarrow+\infty} \sum_{i=1}^{n} \frac{n-i}{n-1} \frac{1}{\sum_{j=1}^{n}\left(v_{i} / v_{j}\right)^{-r}}, \\
& =\frac{n-j}{n-1},
\end{aligned}
$$

where $j$ is the index of maximum $v_{i}$.

Accordingly, combine (27) with (A.10), and the aggregation result is

$$
F_{f}\left(x_{1}, x_{2}, \ldots, x_{n}\right)=\max \left\{x_{i}\right\}=x_{l}
$$

The proof of Theorem 6 is completed.

\section{B. Proof of Theorem 8}

Proof. In (29), let vector $V$ satisfy $v_{1} \geqslant v_{2} \geqslant \cdots \geqslant v_{n}$, which can be written as follows:

$$
\begin{aligned}
W_{\lambda} & =\left(w_{1}, w_{2}, \ldots, w_{n}\right)^{T}, \\
& =\left(\frac{v_{1}^{r}}{\sum_{j=1}^{n} v_{j}^{r}}, \frac{v_{2}^{r}}{\sum_{j=1}^{n} v_{j}^{r}}, \ldots, \frac{v_{n}^{r}}{\sum_{j=1}^{n} v_{j}^{r}}\right)^{T} .
\end{aligned}
$$

From (B.1), the derivative of function $w_{i}$ with variable $r$ is as follows:

$$
\frac{\partial w_{i}}{\partial r}=\frac{v_{i}^{r} \ln v_{i} \sum_{i=1}^{n} v_{i}^{r}-v_{i}^{r} \sum_{i=1}^{n} v_{i}^{r} \ln v_{i}}{\left(\sum_{i=1}^{n} v_{i}^{r}\right)^{2}}
$$

Accordingly, from (28) and (B.2), the derivative of orness $\alpha$ with variable $r$ is formed as follows:

$$
\begin{aligned}
\frac{\partial \alpha}{\partial r}= & \sum_{i=1}^{n} \frac{n-i}{n-1} \frac{\partial w_{i}}{\partial r} \\
= & \sum_{i=1}^{n} \frac{n-i}{n-1} \frac{v_{i}^{r} \ln v_{i} \sum_{i=1}^{n} v_{i}^{r}-v_{i}^{r} \sum_{i=1}^{n} v_{i}^{r} \ln v_{i}}{\left(\sum_{i=1}^{n} v_{i}^{r}\right)^{2}} \\
= & \frac{1}{n-1} \frac{1}{\left(\sum_{i=1}^{n} v_{i}^{r}\right)^{2}} \\
& \times\left[(n-1)\left(0+v_{1}^{r} v_{2}^{r} \ln \frac{v_{1}}{v_{2}}+\cdots+v_{1}^{r} v_{n}^{r} \ln \frac{v_{1}}{v_{n}}\right)\right. \\
& +(n-2)\left(v_{2}^{r} v_{1}^{r} \ln \frac{v_{2}}{v_{1}}+0+\cdots+v_{2}^{r} v_{n}^{r} \ln \frac{v_{2}}{v_{n}}\right) \\
& +(n-3)\left(v_{3}^{r} v_{1}^{r} \ln \frac{v_{3}}{v_{1}}+\cdots\right. \\
& \left.\left.+v_{3}^{r} v_{2}^{r} \ln \frac{v_{3}}{v_{2}}+\cdots+0\right)+\cdots+0\right] \\
& \frac{1}{n-1} \frac{1}{\left(\sum_{i=1}^{n} v_{i}^{r}\right)^{2}} \sum_{i=1}^{n} \sum_{i<j}\left(v_{i}^{r} v_{j}^{r}\right) \frac{v_{i}}{v_{j}} .
\end{aligned}
$$

Since $v_{1} \geqslant v_{2} \geqslant \cdots \geqslant v_{n}$, it is concluded that $\partial \alpha / \partial r>0$. Namely, orness $\alpha$ increases monotonically with parameter $r$. So when $r_{1}>r_{2}$, orness $\left(W_{r_{1}}\right)>$ orness $\left(W_{r_{2}}\right)$.

The proof of Theorem 8 is completed.

\section{Acknowledgment}

The work is supported by the National Natural Science Foundation of China (NSFC) under Project 71171048.

\section{References}

[1] R. R. Yager, "On ordered weighted averaging aggregation operators in multicriteria decision making," IEEE Transactions on Systems, Man and Cybernetics, vol. 18, no. 1, pp. 183-190, 1988.

[2] G. Beliakov, A. Pradera, and T. Calvo, Aggregation Functions: A Guide for Practitioners, Springer, Berlin, Germany, 2007.

[3] A. Emrouznejad, "MP-OWA: the most preferred OWA operator," Knowledge-Based Systems, vol. 21, no. 8, pp. 847-851, 2008.

[4] G. R. Amin and A. Emrouznejad, "Parametric aggregation in ordered weighted averaging," International Journal of Approximate Reasoning, vol. 52, no. 6, pp. 819-827, 2011.

[5] E. Herrera-Viedma, F. Herrera, L. Martínez, J. C. Herrera, and A. G. López, "Incorporating filtering techniques in a fuzzy linguistic multi-agent model for information gathering on the web," Fuzzy Sets and Systems, vol. 148, no. 1, pp. 61-83, 2004.

[6] Y. Liu, "Adaptive self-organized maps based on bidirectional approximate reasoning and its applications to information filtering," Knowledge-Based Systems, vol. 19, no. 8, pp. 719-729, 2006. 
[7] S. Xian, "Fuzzy linguistic induced ordered weighted averaging operator and its application," Journal of Applied Mathematics, vol. 2012, Article ID 210392, 10 pages, 2012.

[8] X. Liu, "Some properties of the weighted OWA operator," IEEE Transactions on Systems, Man, and Cybernetics B, vol. 36, no. 1, pp. 118-127, 2006.

[9] R. R. Yager, "Time series smoothing and OWA aggregation," IEEE Transactions on Fuzzy Systems, vol. 16, no. 4, pp. 994-1007, 2008.

[10] B. S. Ahn and H. Park, "An efficient pruning method for decision alternatives of OWA operators," IEEE Transactions on Fuzzy Systems, vol. 16, no. 6, pp. 1542-1549, 2008.

[11] X. Liu, "Models to determine parameterized ordered weighted averaging operators using optimization criteria," Information Sciences, vol. 190, pp. 27-55, 2012.

[12] Z. Zhang and S. Zhang, "Type-2 fuzzy soft sets and their applications in decision making," Journal of Appllied Mathematics, vol. 2012, Article ID 608681, 35 pages, 2012.

[13] X. Liu, "An orness measure for quasi-arithmetic means," IEEE Transactions on Fuzzy Systems, vol. 14, no. 6, pp. 837-848, 2006.

[14] Z. Gong, L. Li, J. Cao, and F. Zhou, "On additive consistent properties of the intuitionistic fuzzy preference relation," International Journal of Information Technology and Decision Making, vol. 9, no. 6, pp. 1009-1025, 2010.

[15] Z. Wei and H. Jianmin, "Intuitionistic fuzzy normalized weighted bonferroni mean and its application in multicriteria decision making," Journal of Applied Mathematics, vol. 2012, Article ID 136254, 22 pages, 2012.

[16] J. Kacprzyk and S. Zadrony, "Computing with words in intelligent database querying: standalone and Internet-based applications," Information Sciences, vol. 134, no. 1-4, pp. 71-109, 2001.

[17] A. H. Keyhanipour, B. Moshiri, M. Kazemian, M. Piroozmand, and C. Lucas, "Aggregation of web search engines based on users' preferences in WebFusion," Knowledge-Based Systems, vol. 20, no. 4, pp. 321-328, 2007.

[18] E. Herrera-Viedma, O. Cordón, M. Luque, A. G. Lopez, and A. M. Muñoz, "A model of fuzzy linguistic IRS based on multi-granular linguistic information," International Journal of Approximate Reasoning, vol. 34, no. 2-3, pp. 221-239, 2003.

[19] H. Bustince, D. Paternain, B. De Baets et al., "Two methods for image compression/reconstruction using owa operators," in Recent Developments in the Ordered Weighted Averaging Operators: Theory and Practice, R. Yager, J. Kacprzyk, and G. Beliakov, Eds., vol. 265 of Studies in Fuzziness and Soft Computing, pp. 229-253, Springer, Berlin, Germany, 2011.

[20] D. Filev and R. R. Yager, "Analytic properties of maximum entropy OWA operators," Information Sciences, vol. 85, no. 1-3, pp. 11-27, 1995.

[21] R. Fullér and P. Majlender, "On obtaining minimal variability OWA operator weights," Fuzzy Sets and Systems, vol. 136, no. 2, pp. 203-215, 2003.

[22] P. Majlender, "OWA operators with maximal Rényi entropy," Fuzzy Sets and Systems, vol. 155, no. 3, pp. 340-360, 2005.

[23] Y. M. Wang, Y. Luo, and X. Liu, "Two new models for determining OWA operator weights," Computers and Industrial Engineering, vol. 52, no. 2, pp. 203-209, 2007.

[24] D. Filev and R. R. Yager, "On the issue of obtaining OWA operator weights," Fuzzy Sets and Systems, vol. 94, no. 2, pp. 157-169, 1998.

[25] P. Liu and F. Jin, "The trapezoid fuzzy linguistic bonferroni mean operators and their application to multiple attribute decision making," Scientia Iranica, vol. 19, pp. 1947-1959, 2012.
[26] Z. Xu, "Intuitionistic fuzzy aggregation operators," IEEE Transactions on Fuzzy Systems, vol. 15, no. 6, pp. 1179-1187, 2007.

[27] P. Liu, "A weighted aggregation operators multi-attribute group decision-making method based on interval-valued trapezoidal fuzzy numbers," Expert Systems with Applications, vol. 38, no. 1, pp. 1053-1060, 2011.

[28] P. Liu and F. Jin, "Methods for aggregating intuitionistic uncertain linguistic variables and their application to group decision making," Information Sciences, vol. 205, pp. 58-71, 2012.

[29] R. R. Yager, "Families of OWA operators," Fuzzy Sets and Systems, vol. 59, no. 2, pp. 125-148, 1993.

[30] R. R. Yager and D. P. Filev, "Parametrized and-like and or-like own operators," International Journal of General Systems, vol. 22, no. 3, pp. 297-316, 1994.

[31] M. Marimin, M. Umano, I. Hatono, and H. Tamura, "Linguistic labels for expressing fuzzy preference relations in fuzzy group decision making," IEEE Transactions on Systems, Man, and Cybernetics B, vol. 28, no. 2, pp. 205-218, 1998.

[32] M. Marimin, M. Umano, I. Hatono, and H. Tamura, "Hierarchical semi-numeric method for pairwise fuzzy group decision making," IEEE Transactions on Systems, Man, and Cybernetics $B$, vol. 32, no. 5, pp. 691-700, 2002.

[33] J. I. Peláez and J. M. Doña, "Majority additive-ordered weighting averaging: a new neat ordered weighting averaging operator based on the majority process," International Journal of Intelligent Systems, vol. 18, no. 4, pp. 469-481, 2003.

[34] J. I. Peláez and J. M. Doña, "A majority model in group decision making using QMA-OWA operators," International Journal of Intelligent Systems, vol. 21, no. 2, pp. 193-208, 2006.

[35] X. Liu and H. Lou, "Parameterized additive neat OWA operators with different orness levels," International Journal of Intelligent Systems, vol. 21, no. 10, pp. 1045-1072, 2006.

[36] J. Wu, C. Y. Liang, and Y. Q. Huang, "An argument-dependent approach to determining OWA operator weights based on the rule of maximum entropy," International Journal of Intelligent Systems, vol. 22, no. 2, pp. 209-221, 2007.

[37] G. R. Amin and A. Emrouznejad, "Optimizing search engines results using linear programming," Expert Systems with Applications, vol. 38, no. 9, pp. 11534-11537, 2011.

[38] J. M. Merigó and M. Casanovas, "Decision-making with distance measures and induced aggregation operators," Computers and Industrial Engineering, vol. 60, no. 1, pp. 66-76, 2011.

[39] X. Liu, "The orness measures for two compound quasiarithmetic mean aggregation operators," International Journal of Approximate Reasoning, vol. 51, no. 3, pp. 305-334, 2010. 


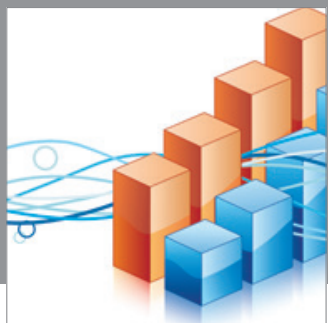

Advances in

Operations Research

mansans

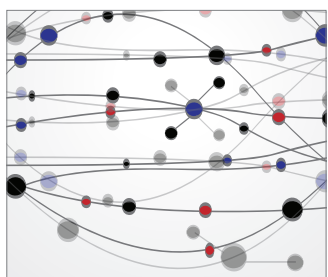

The Scientific World Journal
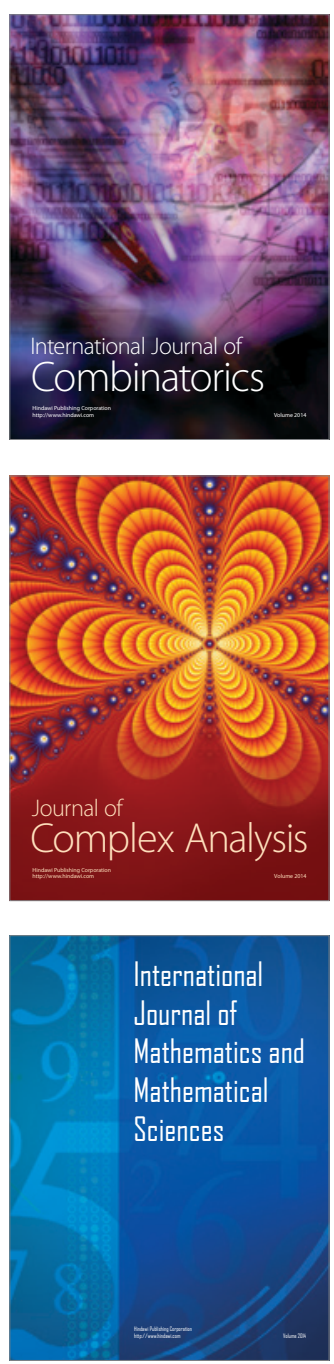
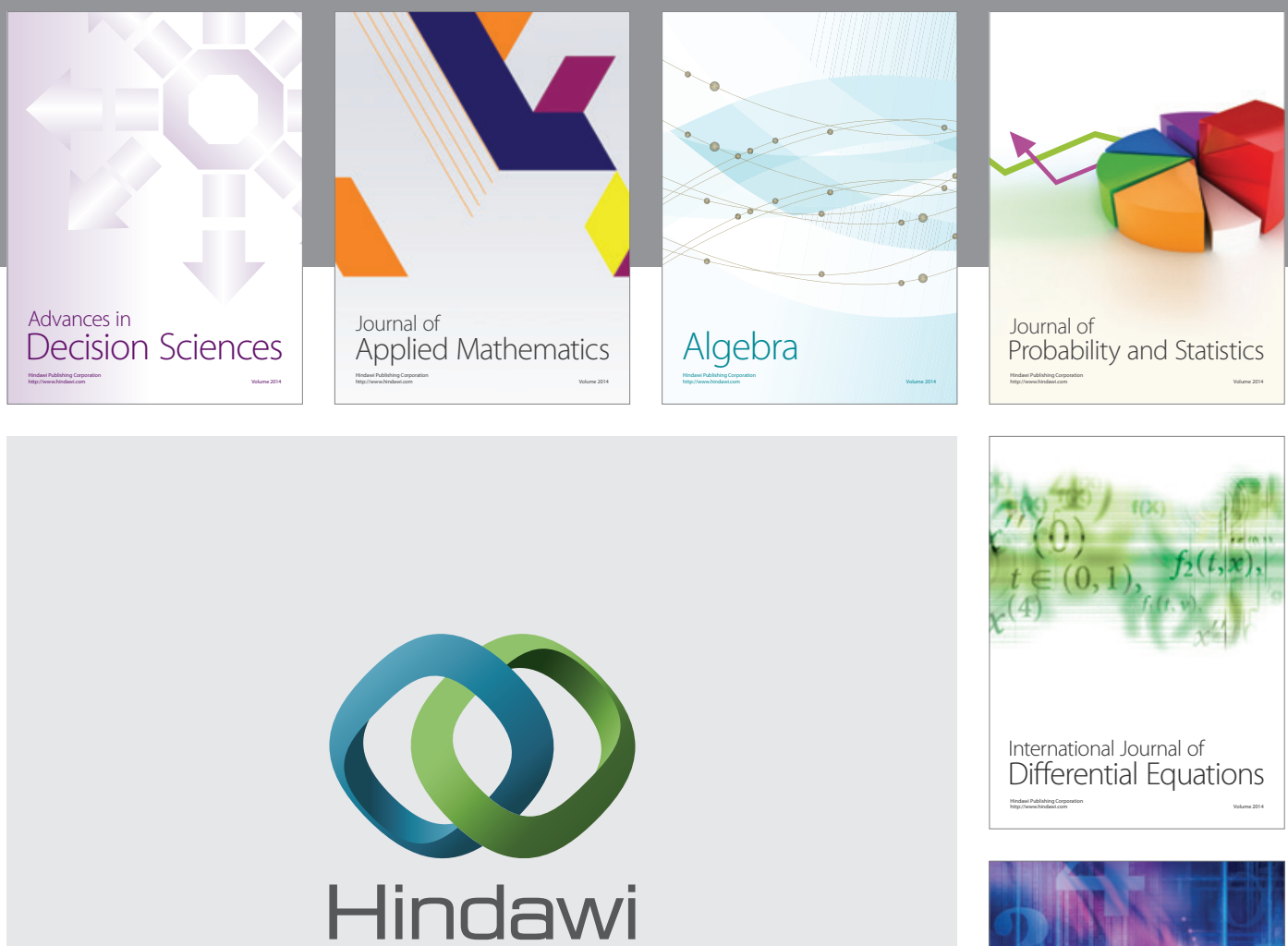

Submit your manuscripts at http://www.hindawi.com
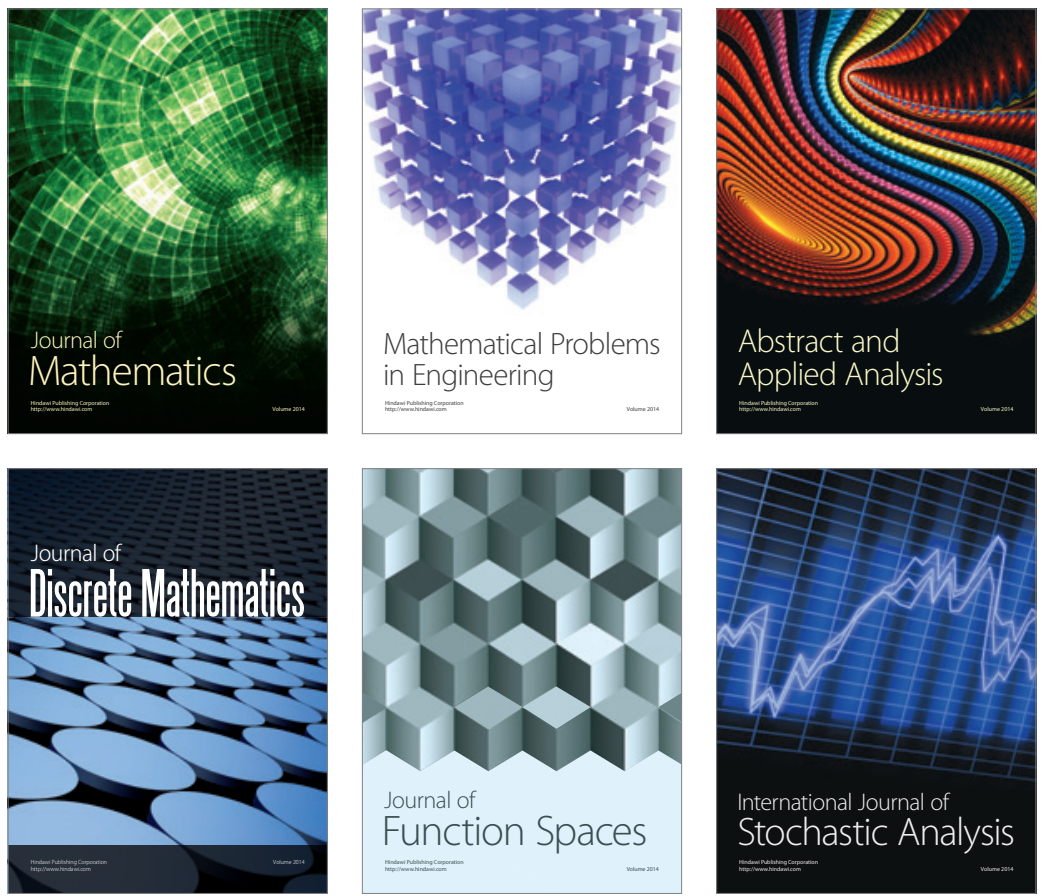

Journal of

Function Spaces

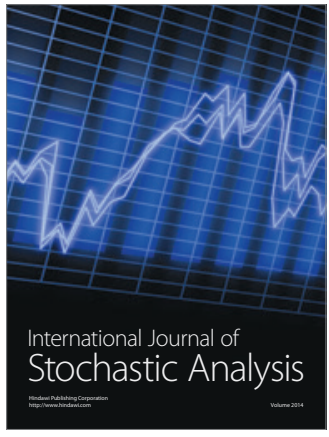

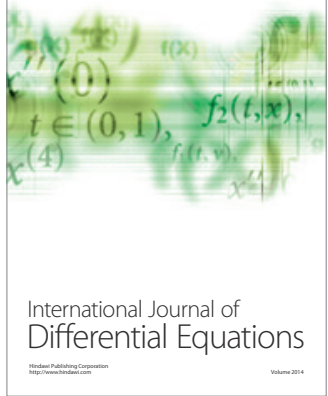
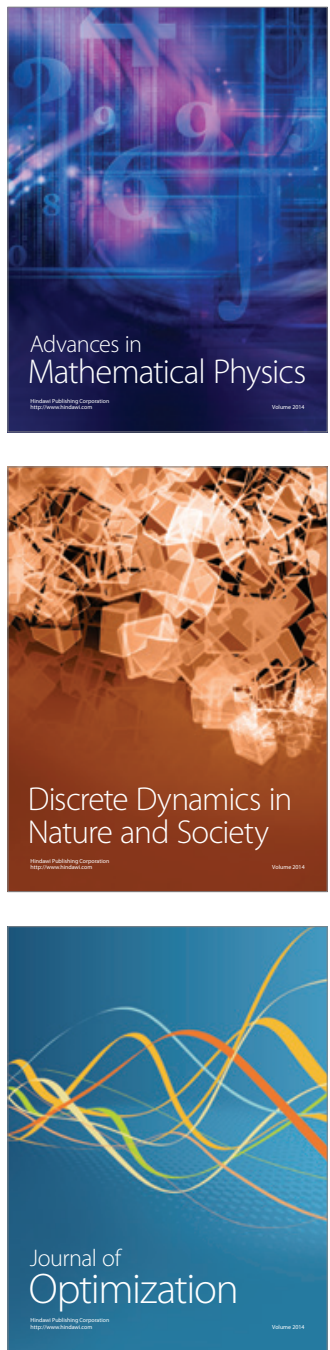